\title{
Causality and Explanation in the Sciences: the Rest of the Best
}

\author{
Bert Leuridan • Erik Weber
}

Received: 10 July 2013/ Accepted: 10 July 2013/Published online: 24 July 2013

(C) Springer Science+Business Media Dordrecht 2013

In 1991 The Pogues, a Celtic punk band, released a compilation album with the well-chosen name The Best of the Pogues. It contained fourteen brilliant songs many of which are still part of our collective memory. Soon after that, they proudly presented another compilation album - this time entitled The Rest of the Best-with songs which were all as splendid as their predecessors.

Twenty years later, in September 2011, philosophers of science, logicians, mathematicians, biologists, social scientists, computer scientists and the like gathered at Ghent University to discuss the relation between causality and explanation: Causality and Explanation in the Sciences (CaEitS2011). ${ }^{1}$ In the course of 3 days, a range of topics were discussed. Different accounts of causality and explanation, such as Jim Woodward's interventionist account, Michael Strevens' kairetic account, and the mechanistic account. The relation between causality, explanation and understanding. The nature and status of causality and explanation in biology, in the social sciences, in medicine, in physics and in mathematics. The relation between causal and constitutive explanation. How causal relations can be discovered and what we can infer from our causal knowledge.

Five of the many brilliant contributions at CaEitS2011 have been published in a special issue of Theoria (vol. 27, no. 2, 2012). If that issue deserves the nickname The Best of CaEitS2011, then the present issue of Erkenntnis can rightly be called The Rest of the Best. We are happy to say that the eight papers below are all as splendid as their predecessors.

\footnotetext{
1 See http://www.caeits2011.ugent.be/.
}

Bert Leuridan is Postdoctoral Fellow of the Fund for Scientific Research - Flanders (FWO)

B. Leuridan $(\bowtie) \cdot$ E. Weber

Centre for Logic and Philosophy of Science, Ghent University, Ghent, Belgium

e-mail: Bert.Leuridan@UGent.be 
We start with the problem of causality and explanation in two specific scientific disciplines: two papers focus on biology, two others on physics. Then we have three papers touching-from different angles-on causality, explanation and mechanisms. The last paper argues against causal pluralism.

In the first paper, "Emergence, closure and inter-level causation in biological systems", Matteo Mossio, Leonardo Bich and Alvaro Moreno advocate the idea that an adequate explanation of biological systems requires appealing to organizational closure as an emergent causal regime. They first develop a theoretical justification of emergence in terms of relatedness, by arguing that configurations, because of the relatedness among their constituents, possess ontologically irreducible properties, providing them with distinctive causal powers. They then focus on those emergent causal powers exerted as constraints, and claim that biological systems crucially differ from other natural systems in that they realize a closure of constraints, i.e. a second-order emergent regime of causation such that the constituents, each of them acting as a constraint, realize a mutual dependence among them, and are collectively able to self-maintain. Lastly, they claim that closure can be justifiably taken as an emergent regime of causation, without admitting that it inherently involves reflexive inter-level causation, which would require to commit to stronger ontological and epistemological assumptions.

Second, Michael Joffe's paper "The concept of causation in biology" sets out to analyze how causation works by focusing on two contrasting branches of biology, to wit epidemiology and physiology. Joffe starts by exploring the specificity of evolved physiological systems, in which evolutionary, developmental and proximal causes all fit together, and the concept of function is meaningful. In contrast, this structure does not apply in epidemiology (or outside biology). Using these two contrasting branches of biology, Joffe examines the role both of mechanism and of difference making in causation. He argues that causation necessarily involves both mechanism and difference making, and that these play complementary roles.

The two papers on causality in physics pull in different directions. Mauricio Suárez argues in the third paper, "Interventions and causality in quantum mechanics", that the Causal Markov Condition (CMC) is in principle applicable to the Einstein-Podolsky-Rosen (EPR) correlations-in line with his earlier defence of the applicability of the Principle of Common Cause to quantum mechanics. He first reviews a contrary claim by Dan Hausman and Jim Woodward who have argued that the CMC is inapplicable to the EPR correlations-i.e. that it neither obtains nor fails-because interventions are unavailable in the case of the EPR experiment. Contra Hausman and Woodward, Suárez urges that whether interventions are available in EPR - and why-is a complex and contextual question that does not have a unique or uniform answer. Instead, he argues that different combinations of causal hypotheses under test, and different interpretations of quantum mechanics, will yield different answers to the question.

In the fourth paper, "A relic of a bygone age? Causation, time symmetry and the directionality argument”, Alexander Reutlinger and Matt Farr start from Russell's conclusion that causation is not part of the fundamental physical description of the world. For Russell, the notion of cause was 'a relic of a bygone age'. Reutlinger and Farr assess one of Russell's arguments for this conclusion: the 'Directionality 
Argument', which holds that the time symmetry of fundamental physics is inconsistent with the time asymmetry of causation. They claim that the coherence and success of the Directionality Argument crucially depends on the proper interpretation of the 'time symmetry' of fundamental physics as it appears in the argument, and offer two alternative interpretations. They argue that: (1) if 'time symmetry' is understood as the time-reversal invariance of physical theories, then the crucial premise of the Directionality Argument should be rejected; and (2) if 'time symmetry' is understood as the temporally bidirectional nomic dependence relations of physical laws, then the crucial premise of the Directionality Argument is far more plausible. They defend the second reading as continuous with Russell's writings, and consider the consequences of the bidirectionality of nomic dependence relations in physics for the metaphysics of causation.

In the fifth paper, "Mechanistic explanation: Integrating the ontic and epistemic", Phyllis Illari scrutinizes the debate between current defenders of ontic explanation (such as Craver) and defenders of epistemic explanation (such as Bechtel) in the context of mechanistic explanation. Illari explores what Bechtel's and Craver's claims mean, and argues that good mechanistic explanations must satisfy both ontic and epistemic normative constraints on what is a good explanation. She argues for ontic constraints by drawing on Craver's work and for epistemic constraints by drawing on Bechtel's work. Along the way, she argues that Bechtel and Craver actually agree with this claim. Then she argues that that we should not take either kind of constraints to be fundamental and closes by considering what remains at stake in making a distinction between ontic and epistemic constraints on mechanistic explanation. She suggests that we should not concentrate on either kind of constraint, to the neglect of the other, arguing for the importance of seeing the relationship as one of integration.

The sixth paper is Jon Williamson's "How can causal explanations explain?" The mechanistic and causal accounts of explanation are often conflated to yield a 'causal-mechanical' account, he argues. His paper prizes them apart and asks: if the mechanistic account is correct, how can causal explanations be explanatory? The answer to this question varies according to how causality itself is understood. According to Williamson, difference-making, mechanistic, dualist and inferentialist accounts of causality all struggle to yield explanatory causal explanations, whereas an epistemic account of causality is more promising in this regard.

The seventh paper is Petri Ylikoski's "Causal and constitutive explanation compared". According to Ylikoski, these two types of explanation have different kinds of explananda and they track different sorts of dependencies. Constitutive explanations do not address events or behaviors, but causal capacities. While there are some interesting relations between building and causal manipulation, causation and constitution are not to be confused. However, despite their metaphysical differences, the same key ideas about explanation largely apply to both. Causal and constitutive explanations face similar challenges (such as the problems of relevance and explanatory regress) and both are in the business of mapping networks of counterfactual dependence-i.e. mechanisms-although the relevant counterfactuals are of a different sort. In the final section Ylikoski discusses the issue of developmental explanation and argues that developmental explanations deserve 
their own place in the taxonomy of explanations, although ultimately developmental dependencies can be analyzed as combinations of causal and constitutive dependencies. Hence, causal and constitutive explanation are distinct, but not always completely separate forms of explanation.

Finally, Michael Strevens' "Causality reunified" opposes to causal pluralism. Hall has recently argued that there are two concepts of causality, picking out two different kinds of causal relation. McGrath, and Hitchcock and Knobe, have recently argued that the facts about causality depend on what counts as a "default" or "normal" state, or even on the moral facts. In the light of these claims you might be tempted to agree with Skyrms that causal relations constitute, metaphysically speaking, an "amiable jumble", or with Cartwright that 'causation', though a single word, encompasses many different kinds of things. Strevens argues however, drawing on his work on explanation, that the evidence adduced in support of causal pluralism can be accommodated easily by a unified theory of causality - a theory according to which all singular causal claims concern the same fundamental causal network.

Causality and Explanation in the Sciences (CaEitS2011) was the sixth episode in the Causality in the Sciences series of conferences which originated at the University of Kent. ${ }^{2}$ Other conferences have focussed on causality and probability in the sciences, on mechanisms and causality in the sciences, on causality in the biomedical and the social sciences, or on evidence and causality in the sciences. In July 2013, the Paris-Sorbonne University has hosted Causality and Experimentation in the Sciences. Papers presented at the previous CitS conferences have resulted in interesting publications. So far, two book volumes have appeared: Federica Russo \& Jon Williamson (eds.), Causality and Probability in the Sciences (London: College Publications, 2007), and Phyllis McKay Illari, Federica Russo \& Jon Williamson (eds.), Causation in the Sciences (Oxford: Oxford University Press, 2011). Moreover, special issues on causality in the sciences have been published, or will be published, in Studies in the History and Philosophy of Science (vol. 43, no. 4, 2012), in Theoria (vol. 27, no. 2, 2012), or in Topoi.

To conclude, we would like to thank a number of people and organizations without the help and support of whom CaEitS2011 would not have taken place. First of all, we thank the other members of the then CitS steering committee (Phyllis Illari, Julian Reiss, Federica Russo and Jon Williamson) and Leen De Vreese for their help in carefully selecting the speakers of our conference. Second, we thank our keynote speakers, Nancy Cartwright, Daniel Little, Henk de Regt, Mauricio Suárez and Michael Strevens for their contributions at the conference. Several members of the Centre for Logic and Philosophy of Science of Ghent University helped us enormously with the practical organization. Financial support was provided by the Fund for Scientific Research-Flanders (FWO), the Faculty of Arts \& Philosophy of Ghent University, and the Special Research Fund (BOF) of Ghent University.

We would also like to thank a number of people for their help with this special issue: the contributing authors for their interesting submissions, the reviewers for

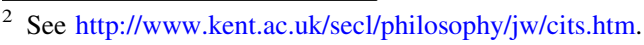


their most helpful reports, and last but not least, Hannes Leitgeb for hospitably welcoming us in Erkenntnis and for his tremendous support during the whole reviewing and editing process. 